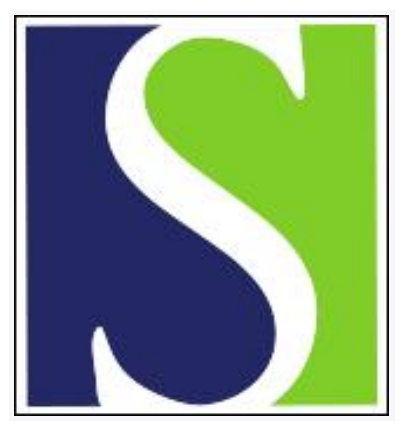

Scand J Work Environ Health 2013;39(3):295-301

https://doi.org/10.5271/sjweh.3271

Published online: 13 Jan 2012, Issue date: 01 May 2013

\title{
Occupational exposure to particles and incidence of stroke
}

by Sjögren B, Lönn M, Fremling K, Feychting M, Nise G, Kauppinen T, Plato N, Wiebert P, Gustavsson P

Affiliation: Work Environment Toxicology Institute of Environmental Medicine, Karolinska Institutet, SE-171 77 Stockholm, Sweden. bengt.sjogren@ki.se

Refers to the following text of the Journal: 2008;34(1):40-47

The following article refers to this text: 2015;41(3):280-287

Key terms: gender; hemorrhagic stroke; ischemic stroke; occupational exposure; occupational exposure; particle; sex; stroke

This article in PubMed: www.ncbi.nlm.nih.gov/pubmed/22241632 


\title{
Occupational exposure to particles and incidence of stroke
}

\author{
by Bengt Sjögren, PhD, ${ }^{1}$ Maria Lönn, MSc, ${ }^{1}$ Karin Fremling MSc, ${ }^{1}$ Maria Feychting, PhD, ${ }^{1}$ Gun Nise, \\ PhD, ${ }^{2,3}$ Timo Kauppinen, PhD, ${ }^{4}$ Nils Plato, PhD, ${ }^{1}$ Pernilla Wiebert, PhD, ${ }^{1}$ Per Gustavsson PhD ${ }^{1}$
}

\begin{abstract}
Sjögren B, Lönn M, Fremling K, Feychting M, Nise G, Kauppinen T, Plato N, Wiebert P, Gustavsson P. Occupational exposure to particles and incidence of stroke. Scand J Work Environ Health. 2013;39(3):295-301. doi:10.5271/ sjweh.3271
\end{abstract}

Objectives This paper aims to investigate the relation between occupational exposure to particles, particle size, and the incidence of ischemic and hemorrhagic stroke.

Methods The cohort included all manual workers identified from the Swedish National Census in 1980, who were alive as of 1 January 1987. First time events of ischemic or hemorrhagic stroke during the period 1987-2005 were identified through linkage to the Hospital Discharge Register and the National Cause of Death Register. A job-exposure matrix for exposure to small $(<1 \mu \mathrm{m})$ and large $(>1 \mu \mathrm{m})$ particles was developed and applied. Hazard ratios (HR) were estimated by Cox regression with adjustment for age, socioeconomic group, and residential area.

Results Increased HR of ischemic stroke were found among both women and men occupationally exposed to small as well as large particles for $\geq 5$ years. The risks were higher for workers exposed for $\geq 5$ years compared to "ever exposed" participants indicating a dose-response relationship, but no trend with exposure intensity was observed. A tentative association between particle exposure and hemorrhagic stroke was also found.

Conclusions Occupational exposure to small and large particles was associated with increased risks of ischemic stroke. Further studies are needed to explore the relationships between exposure to different types of particles and various doses and the occurrence of stroke among women as well as men.

Key terms gender; hemorrhagic stroke; ischemic stroke, sex.

Stroke is a term that signifies the abrupt impairment of brain function caused by a variety of changes involving one or several cerebral blood vessels. The annual estimated number of first-ever stroke in Sweden is around 30000 and the mean age is around 73 years for men and 77 years for women (1). Between 1989-2000, the incidence of stroke increased by $19 \%$ and $33 \%$ among men and women, respectively. The largest increase was observed among those $<60$ years (2). Stroke is the third most common cause of death in Sweden (1), and stroke mortality appears to decline (2).

Approximately $85 \%$ of all strokes are caused by diminished blood flow (ischemic stroke) and the remaining $15 \%$ include hemorrhage into the brain tissue and the surrounding subarachnoid space. Hypertension, smoking, diabetes, and atrial fibrillation are well-established risk factors for stroke (3).

Particulate urban air pollutants have been associated with stroke in some investigations (4-6). Some studies have also observed an increased risk of cerebrovascular disease among potentially particle-exposed workers such as building and construction workers, chimney sweeps (7), aluminum potroom workers (8), and army cooks (9), but other studies have not revealed such associations (10).

This study is a part of the PARticles and Cardio- and Cerebrovascular diseases (PARCC) project. The aim of the present study was to investigate the association between occupational exposure to particles, particle size, and the incidence of stroke among women and men.

1 Institute of Environmental Medicine, Karolinska Institutet, Stockholm, Sweden.

2 Department of Public Health Sciences, Karolinska Institutet, Stockholm, Sweden.

3 Deceased.

4 Finnish Institute of Occupational Health (FIOH), Helsinki, Finland.

Correspondence to: Bengt Sjögren, Work Environment Toxicology Institute of Environmental Medicine, Karolinska Institutet, SE-171 77 Stockholm, Sweden. [E-mail: bengt.sjogren@ki.se] 


\section{Methods}

\section{Study base}

The cohort comprises all manual workers in the Swedish National Census in 1980, who were alive as of 1 January 1987, in total 984040 men and 741631 women. The cohort was restricted to manual workers, including skilled and unskilled manual workers in the production and service sectors, in order to limit potential confounding from smoking and other lifestyle factors. Thus, white-collar workers, professionals, self-employed, and farmers were not included. First time events of ischemic stroke or cerebral infarction [International Classification of Diseases, $9^{\text {th }}$ Revision (ICD-9) code 434 or $10^{\text {th }}$ Revision (ICD-10) code I63] and hemorrhagic stroke (ICD-9 code 431 or ICD-10 code I61) during the period 1987-2005 were identified through linkage to the nationwide Hospital Discharge Register and the National Cause of Death Register. Only first-time events of cerebral infarction or hemorrhagic stroke were considered in the respective analysis. We also recorded diagnoses of cardiac arrhythmias (ICD-9 code 427 or ICD-10 code I47-I49), hypertension (ICD-9 code 401-405 or ICD-10 code I10-I15), and diabetes mellitus (ICD-9 code 250 or ICD-10 code E10-E14) for later scrutinizing groups with increased susceptibility of developing stroke.

The Regional Ethical Review Board in Stockholm, Sweden, approved the study.

\section{Exposure assessment}

Information on occupation, socioeconomic status, and residential population density was obtained from the population censuses in 1980, 1985, and 1990. The censuses provide occupational codes according to the Nordic adaption of the International Standard Classification of Occupations [Nordisk yrkesklassificering (Nordic Occupational Classification) 1978] (11). A job-exposure matrix (PARCC-JEM) was developed by experienced occupational hygienists by combining relevant occupational particle exposure information from a Swedish JEM developed for the Nordic Occupational Cancer Study (NOCCA) (12) and an airway irritantJEM (13). The estimated levels in the PARCC-JEM are based on exposure measurements from Sweden when available and otherwise from other Nordic countries. Twelve groups of particle exposure were used from the NOCCA-JEM and seven from the airway irritantJEM. Together these 19 groups of particle exposure covered the occupational particle exposure of interest, except for cooking fumes. We therefore assessed exposure to cooking fumes in all occupations in the JEM. The PARCC-JEM thus includes 20 groups of particle exposure and about 300 occupations (13). Each cell of the JEM shows the proportion of exposed workers and the exposure level (air concentration) for each particle group. The JEM is time-specific and covers two time periods: $1975-1984$ and 1985-1994.

Exposure levels were assessed using a semi-quantitative scale with four categories, expressed as proportions of the Swedish occupational exposure limits (Swedish Work Environment Authority Regulations) (14) $<1 / 30$ (no exposure), 1/30-1/10 (low exposure level), 1/10-1/3 (medium exposure level) and $>1 / 3$ (high exposure level). To achieve as great a contrast in exposure as possible between exposed and unexposed workers, we defined an occupation as exposed only if there was a high prevalence of particle exposure ( $\geq 80 \%$ exposed in the occupation). To investigate the influence of particle size, the particle exposures in the JEM were classified as small or large particle size $(<1 \mu \mathrm{m}$ and $>1 \mu \mathrm{m}$, respectively). Subjects in particle-exposed occupations with an exposure prevalence $<80 \%$ were excluded from the analyses.

The PARCC-JEM included 104 occupations with particle exposure, but only 31 occupations were associated with medium- and high-particle exposure and a high prevalence $(\geq 80 \%)$. In 50 occupations, no exposure assessment was feasible, mostly due to undefined working tasks or heterogeneous group of tasks with different exposures. Unexposed occupations were defined as occupations without exposure to any of the particles in the JEM, totaling 128 occupations. In total, 494674 men and 488735 women were included in the analyses for ischemic stroke, and 494737 men, and 488769 women were included in the analyses for hemorrhagic stroke. Ischemic stroke cases diagnosed prior to the start of follow-up were excluded from analysis of ischemic stroke, and hemorrhagic stroke cases diagnosed prior to start of follow-up were excluded from analysis of hemorrhagic stroke.

\section{Statistical analysis}

Subjects were considered to be under risk from 1 January 1987 until first episode of ischemic or hemorrhagic stroke, death, emigration, or 31 December 2005, whichever occurred first. In case of immigration of a formerly emigrated person in the study base, the person contributed with person-years from the immigration date until any of the events mentioned above occurred.

Exposure status was determined from the 1980 census and was updated according to the 1985 and 1990 censuses if exposure status had changed. The association between particle exposure and the outcome was estimated through Cox proportional hazards modeling. Hazard ratios (HR) for men and women are presented with 95\% confidence intervals $(95 \% \mathrm{CI})$. All risk estimates were adjusted for age (continuous variable), socioeconomic group (4 categories: skilled or unskilled manual 
workers in production or service sectors, respectively) and residential population density ( 5 categories based on population density: $<200,200-4999,5000-19999$, $20000-99999$, and $\geq 100000$ inhabitants) as a proxy for environmental air pollution. When the outcome of exposure to small particles was studied, the calculations were adjusted for exposure to large particles and vice versa.

To assess the effect of exposure duration, we restricted one analysis to subjects with exposure to particles in at least two consecutive censuses, which are five years apart (ie, subjects who had the same occupational code, exposure status, and socioeconomic position in at least two consecutive censuses in 1980, 1985, and 1990). The corresponding restrictions were also applied to the unexposed subjects.

A subject may be unexposed and belong to the unexposed group for some period of time and later become exposed and consequently belong to an exposed group for the rest of the follow-up period. Subjects may be occupationally exposed to several agents and may, therefore, have been exposed to both large and small particles.

\section{Results}

In total, the cohorts comprised 983409 and 983506 participants for ischemic stroke and hemorrhagic stroke, respectively. Detailed characteristics of the cohort used for analyzing ischemic stroke are described in table 1. The characteristics of the cohort used for analyzing hemorrhagic stroke were similar and are not presented.

During the follow up period 1987-2005, 43080 first-time events of ischemic stroke were registered, of which 23437 occurred among men. The total number of registered hemorrhagic strokes was 9070, 5092 of which occurred among men.

Women and men exposed to small or large particles for $\geq 5$ years showed an increased risk of ischemic stroke, after adjusting for age, socioeconomic status, and residential population density (table 2). HR for "ever exposed" to small or large particles were slightly lower. The crude HR (without adjustment for socioeconomic status and residential population density) were slightly lower for men exposed to small particles (HR 1.04, 95\% CI 1.01-1.08) and large particles (HR 0.97, 95\% CI 0.94-1.01) (data not shown). The crude estimates were almost the same as the adjusted for women exposed to small particles $(1.00,95 \%$ CI $0.95-1.05)$ and large particles $(1.11,95 \%$ CI $1.08-$ 1.14). Workers exposed at two consecutive censuses, five years apart, had higher HR than "ever exposed" workers. There were no signs of a positive trend with exposure intensity, comparing HR among the low-exposed with the medium- and high-exposed, neither among men nor women exposed to small or large particles.
Table 1. Characteristics of the cohort studying ischemic stroke comprising 983409 participants. [SD=standard deviation.]

\begin{tabular}{|c|c|c|c|c|}
\hline Characteristics & $\mathrm{N}$ & $\%$ & Mean & SD \\
\hline Females & 488735 & 49.7 & & \\
\hline Males & 494674 & 50.3 & & \\
\hline Age at entrance (years) & & & 45.5 & 12.6 \\
\hline \multicolumn{5}{|l|}{ Exposure to particles } \\
\hline Unexposed & 328600 & 33.4 & & \\
\hline $\begin{array}{l}\text { Exposure to small particles } \\
\text { (low-, medium- and high-exposure) }\end{array}$ & 157230 & 16.0 & & \\
\hline $\begin{array}{l}\text { Exposure to large particles } \\
\text { (low-, medium- and high-exposure) }\end{array}$ & 570522 & 58.0 & & \\
\hline \multicolumn{5}{|l|}{ Socioeconomic groups } \\
\hline $\begin{array}{l}\text { Skilled manual workers in } \\
\text { production sectors }\end{array}$ & 233740 & 23.8 & & \\
\hline $\begin{array}{l}\text { Skilled manual workers in service } \\
\text { sectors }\end{array}$ & 89419 & 9.1 & & \\
\hline $\begin{array}{l}\text { Unskilled manual workers in } \\
\text { production sectors }\end{array}$ & 199707 & 20.3 & & \\
\hline $\begin{array}{l}\text { Unskilled manual workers in } \\
\text { service sectors }\end{array}$ & 460543 & 46.8 & & \\
\hline \multicolumn{5}{|l|}{ Residential population densities } \\
\hline Large cities $>100000$ inhabitants & 170642 & 17.4 & & \\
\hline Cities 20 000-99 999 inhabitants & 235679 & 24.0 & & \\
\hline Villages 5000-19 999 inhabitants & 208217 & 21.2 & & \\
\hline Villages 200-4999 inhabitants & 208737 & 21.2 & & \\
\hline Villages $<200$ inhabitants & 160134 & 16.3 & & \\
\hline \multicolumn{5}{|l|}{ Registered other diseases } \\
\hline Cardiac arrhythmias & 63069 & 6.4 & & \\
\hline Hypertension & 88294 & 9.0 & & \\
\hline Diabetes mellitus & 49852 & 5.1 & & \\
\hline
\end{tabular}

Women "ever exposed" to large particles showed an increased risk of hemorrhagic stroke (table 3). Men and women exposed for $\geq 5$ years exhibited higher HR regarding both small and large particles than the estimates for "ever exposed" workers. However, no positive trends were observed when comparing low-exposed with medium- and high-exposed workers.

This presentation of exposed and unexposed occupations is solely based on the information from the national census in 1980. The most common occupations among unexposed women were nurse assistants (41\%) and shop assistants (28\%). Among women exposed to low levels of small particles, the dominant occupations were waitresses and head waitresses (97\%) and among medium- and highexposed women, welders and gas-cutters (73\%) and metal furnace operators (22\%). Among women exposed to low levels of large particles, the dominant occupation was cleaners (96\%) and among medium- and high-exposed women, the dominant occupations were engineering workers $(25 \%)$ and tailors and seamstresses $(22 \%)$.

The most common occupations among unexposed men were shop (37\%) and nurse assistants (16\%) and attendants in mental care (14\%). Among men exposed to low levels of small particles, the dominant occupations were machine drivers $(73 \%)$ and waiters and head waiters $(24 \%)$ and among medium- and high-exposed men, the dominant occupations were welders and gas-cutters 
Table 2. Exposed and unexposed cases (N), hazard ratios (HR) and 95\% confidence intervals (95\% $\mathrm{Cl})$ for ischemic stroke among workers exposed to small $(<1 \mu \mathrm{m})$ and large $(>1 \mu \mathrm{m})$ particles, respectively.

\begin{tabular}{|c|c|c|c|c|c|c|}
\hline & \multicolumn{3}{|c|}{ Men } & \multicolumn{3}{|c|}{ Women } \\
\hline & $\mathrm{N}$ & $H R^{a}$ & $95 \% \mathrm{Cl}$ & $\mathrm{N}$ & $\mathrm{HR}^{\mathrm{a}}$ & $95 \% \mathrm{Cl}$ \\
\hline \multicolumn{7}{|l|}{ Ever exposed } \\
\hline Unexposed & 1640 & 1.00 & .. & 9751 & 1.00 & .. \\
\hline Small particles & 4719 & 1.06 & $1.02-1.10$ & 1563 & 1.00 & $0.95-1.05$ \\
\hline Large particles & 19623 & 1.05 & $1.00-1.11$ & 8478 & 1.11 & $1.07-1.15$ \\
\hline Small particles (low-exposed) & 2554 & 1.09 & $1.04-1.15$ & 1435 & 0.98 & $0.93-1.04$ \\
\hline Small particles (medium- and high-exposed) & 2165 & 1.02 & $0.98-1.07$ & 128 & 1.20 & $1.01-1.44$ \\
\hline Large particles (low-exposed) & 2673 & 1.10 & $1.05-1.16$ & 5242 & 1.11 & $1.07-1.14$ \\
\hline Large particles (medium- and high-exposed) & 16950 & 1.05 & $0.99-1.10$ & 3236 & 1.10 & $1.03-1.18$ \\
\hline \multicolumn{7}{|l|}{ Exposed for $\geq 5$ years } \\
\hline Unexposed & 905 & 1.00 & & 5094 & 1.00 & .. \\
\hline Small particles & 1986 & 1.18 & $1.09-1.27$ & 198 & 1.19 & $1.03-1.38$ \\
\hline Large particles & 5464 & 1.10 & $1.02-1.19$ & 3112 & 1.12 & $1.07-1.18$ \\
\hline
\end{tabular}

${ }^{a}$ Adjusted for age, socioeconomic groups, and residential population density

Table 3. Exposed and unexposed cases (N), hazard ratios (HR) and $95 \%$ confidence intervals $(95 \% \mathrm{Cl})$ for hemorrhagic stroke among workers exposed to small $(<1 \mu \mathrm{m})$ and large $(>1 \mu \mathrm{m})$ particles, respectively.

\begin{tabular}{|c|c|c|c|c|c|c|}
\hline & \multicolumn{3}{|c|}{ Men } & \multicolumn{3}{|c|}{ Women } \\
\hline & $\mathrm{N}$ & $H R^{a}$ & $95 \% \mathrm{Cl}$ & $\mathrm{N}$ & $\mathrm{HR}^{\mathrm{a}}$ & $95 \% \mathrm{Cl}$ \\
\hline \multicolumn{7}{|l|}{ Ever exposed } \\
\hline Unexposed & 361 & 1.00 & .. & 1939 & 1.00 & .. \\
\hline Small particles & 1089 & 1.04 & $0.97-1.13$ & 333 & 1.11 & $0.99-1.25$ \\
\hline Large particles & 4208 & 1.02 & $0.92-1.13$ & 1742 & 1.15 & $1.07-1.24$ \\
\hline Small particles (low-exposed) & 619 & 1.11 & $1.00-1.23$ & 305 & 1.10 & $0.98-1.24$ \\
\hline Small particles (medium- and high-exposed) & 470 & 0.97 & $0.88-1.07$ & 28 & 1.19 & $0.82-1.75$ \\
\hline Large particles (low-exposed) & 597 & 1.04 & $0.93-1.16$ & 1081 & 1.14 & $1.05-1.23$ \\
\hline Large particles (medium- and high-exposed) & 3611 & 1.00 & $0.89-1.12$ & 661 & 1.13 & $0.97-1.31$ \\
\hline \multicolumn{7}{|l|}{ Exposed for $\geq 5$ years } \\
\hline Unexposed & 194 & 1.00 & .. & 1092 & 1.00 & .. \\
\hline Small particles & 477 & 1.24 & $1.06-1.46$ & 43 & 1.34 & $0.99-1.83$ \\
\hline Large particles & 1200 & 1.13 & $0.96-1.33$ & 698 & 1.23 & $1.11-1.37$ \\
\hline
\end{tabular}

${ }^{a}$ Adjusted for age, socioeconomic groups, and residential population density

$(71 \%)$ and metal furnace operators $(16 \%)$. Among men exposed to low levels of large particles, the dominant occupations were machine drivers $(33 \%)$ and cleaners (29\%) and among medium- and high-exposed men, the dominant occupations were engineering workers $(25 \%)$, construction carpenters $(16 \%)$, construction workers $(11 \%)$ and welders and gas-cutters $(10 \%)$. Consequently machine drivers, welders, and gas-cutters were exposed to both small and large particles.

\section{Discussion}

Our main finding was an increased risk of ischemic stroke among women as well as men occupationally exposed to small or large particles for $\geq 5$ years. Findings for hemor- rhagic stroke were similar, but of borderline statistical significance.

The key strength of the present study is the inclusion of the entire Swedish population of occupationally active manual workers. The long tradition of population registration in Sweden makes it possible to perform high quality epidemiological studies, through linkage of several registers by the unique personal identity number. Exposure information was derived from census registers and a JEM, and exposure data were thus collected independently of disease status, which prevents reporting bias. The outcome was identified through the nationwide Cause of Death Register and the Hospital Discharge Register. Considering that stroke hospitalization rate has been reported to be $>90 \%$ from different parts of Sweden (2), these two sources are likely to cover well over $90 \%$ of the cases. 
A JEM is a powerful tool and a relatively cost- and time-efficient method compared to other methods of exposure assessment. However, as well as other methods of exposure assessment, JEM will result in some misclassification of exposure. Furthermore, exposure assessment in this study was based on occupations registered in five-year intervals and consequently not representative of the entire work history for most subjects. However, misclassification of exposure in our study is non-differential and will bias the HR towards the null. JEM are optimal when specificity is favored over sensitivity. This study aimed towards a high specificity as a job was classified as exposed only if the probability of exposure was $\geq 80 \%$.

The level of exposure was based on Swedish occupational exposure limits in order to account for the toxic effect of each type of particle. This approach would not be appropriate if the biological effect was solely related to an unspecific particle effect. However, the use of absolute weight $\left(\mathrm{mg} / \mathrm{m}^{3}\right)$ to classify intensity would underestimate the effect of inhaling particles of small size and was not considered an appropriate metric when comparing the effects of small versus large particles.

Between 1989-2000, the incidence of stroke increased by 33\% among women and 19\% among men in Sweden and the largest increase was observed among those under 60 years (2). Among both women and men, socioeconomic status is related to stroke $(15,16)$. In a metaanalysis, when compared to the highest socioeconomic category, the HR for the lowest was 1.67 (95\% CI 1.461.91). When adjusting for classical risk factors (such as hypertension, smoking, diabetes, obesity, alcohol, and physical activity), the HR decreased to 1.31 (95\% CI 1.16-1.48) (15). In the present study, socioeconomic confounding was reduced by restricting the cohort to skilled and unskilled manual workers. Further, adjustment for differences regarding socioeconomic subgroups and residential population densities changed the HR only slightly. Consequently, we assume that most other classical risk factors (such as smoking, obesity, and physical activity) are taken into account by the restriction and adjustment. However, residual confounding cannot be ruled out since female metal workers and male metal and construction workers are compared with nurse and shop assistants. The potential bias from the so-called "healthy worker effect" that frequently affects occupational cohort studies and causes an underestimation of the occupational risks (17) was reduced since the study base was limited to occupationally active persons.

To further evaluate the impact of possible differences in smoking habits between socioeconomic groups, information from the Swedish national public health surveys of 2005-2010 was utilized. The surveys comprise a total of 20000 randomly selected people each year, aged 16-84 years, and gives information on the mean percentage of daily smokers among women and men in different socioeconomic groups. There are small differences regarding smoking habits between the socioeconomic groups of interest in this study. Among women, the proportion of daily smokers varied between $24.6 \%$ (unskilled workers in production sectors) and $20.6 \%$ (skilled workers in service sectors) and between $18.9 \%$ (skilled workers in service sectors) and 15\% (skilled workers in production sectors) among men (18). Smoking increases the risk of stroke to 1.8 (3) and a smoking prevalence of $24.6 \%$ compared with $20.6 \%$ among females will increase the risk of stroke to 1.03, according to Axelson \& Steenland (19). Based on these estimates, smoking will unlikely contribute to a major change in HR for workers exposed to particles. However, residual confounding cannot be ruled out as smoking habits may be different in major subgroups of male and female workers.

The American Heart Association reviewed the literature and presented a statement in 2010 regarding particulate matter air pollution and cardiovascular disease. They summarized the epidemiological evidence of the cardiovascular effects of $\mathrm{PM}_{2.5}$ or traffic- or combustion-related air pollution exposure at ambient levels. There was moderate overall epidemiological evidence for an association between short-term exposure (days) and ischemic stroke. The epidemiological evidence was limited for an association between longer-term exposure (months to years) and ischemic stroke (20). However, a cohort based on the Women's Health Initiative Observational Study showed a relationship between long-term exposure to air pollution and stroke among women (5). Brook and coworkers (20) found strong evidence for inhaled $\mathrm{PM}_{2.5}$ or traffic- or combustion-related air pollution to start a systemic proinflammatory response that will increase blood coagulation. Increased blood coagulation may ultimately cause an ischemic stroke. Brook also suggests that short-term exposure to $\mathrm{PM}_{2.5}$ or diesel exhaust is capable in certain circumstances of rapidly raising blood pressure (20), and hypertension is one of the most important risk factors for hemorrhagic stroke (3). Thus, there are mechanistic links between airborne particles and the occurrence of ischemic as well as hemorrhagic stroke. However, the epidemiological evidence is stronger between inhalation of airborne particles and the occurrence of ischemic stroke than between particles and hemorrhagic stroke. Brook and other researchers also raised the question whether women are at higher risk than men $(20,21)$.

Some previous occupational studies have presented an increased risk of cerebrovascular disease among potentially particle-exposed male workers, such as building and construction workers, chimney sweeps (7), welders (22), aluminum potroom workers (8), and army cooks (9). An increased risk of cerebrovascular disease was also observed among female Finnish building maintenance workers and cleaners (7). Thus, previous 
investigations have mainly observed increased risk of cerebrovascular disease among men.

Low job control has been associated with an increased risk for stroke among men and women. The relationship was stronger for intracerebral hemorrhage compared to brain infarction after adjusting for age, work hours, education, marital status, and income (23). There was a significant relation between low job control and hemorrhagic stroke among female lower class nonmanual workers (24). However, in another study, low job control was related not to hemorrhagic stroke but rather ischemic stroke among women (HR 1.4, $95 \%$ CI 0.9-2.4) (16). We have no information on job control in this study. In a previous Swedish questionnaire study performed during 1997 and 1999 (25), increased workpace was surveyed among workers who worked in the same occupation during the last five years. Workers in non-particulate-exposed occupations had more often experienced an increased workpace during the last five years compared to particulate-exposed workers. This was reported by $66 \%$ of customer service workers (including shop assistants) and $72 \%$ of health and social care workers, which include nurse assistants and attendants in mental care. Among the particleexposed group of restaurant workers, $63 \%$ experienced increased workpace, $65 \%$ of machine drivers, and $55 \%$ of machine operators and assembly workers (including engineering workers), metal workers (including welders) and cleaners experienced increased workpace. Among construction workers and carpenters, the proportion was $54 \%$ and $48 \%$, respectively. Thus it seems that reported experience of increasing workpace may be lower among particle-exposed compared to unexposed workers in our study.

A register study based on longitudinal data on Finnish men between 1981-1994 indicated an increased mortality by cerebrovascular diseases caused by shift work. The rate ratios for 2- and 3-shift was 1.19 (95\% CI 1.011.39 ) and 1.06 (95\% CI 0.86-1.31), respectively (26). In the present study, we have no information on shift work among exposed and unexposed workers. However, in the Swedish survey performed during 1997-1999 (25), workers in non-particulate exposed occupations often generally had working hours other than day work compared to particulate-exposed workers. These working hours included evening or night work, or alternating or rotating shifts. The percentage of non-daytime work was 36\% among customer service workers (including shop assistants) and 59\% among health and social care workers, which include nurse assistants and attendants in mental care. Among the particle-exposed groups of restaurant workers, machine operators, and assembly workers (including engineering workers), $42 \%$ worked non-daytime work. Other particle-exposed occupations had lower proportions of non-daytime work such as metal workers, including welders (17\%), machine drivers $(30 \%)$, cleaners $(14 \%)$, and construction workers and carpenters $(2 \%)$. Thus it seems unlikely that shift work can explain the observed difference regarding stroke between particle- and unexposed workers in our study.

\section{Concluding remarks}

The main finding of this study was an increased risk of ischemic stroke among women as well as men occupationally exposed to small or large particles for $\geq 5$ years. A positive trend was noted with exposure duration but not with exposure intensity. The relationship between particle exposure and hemorrhagic stroke was tentative and not as clear as for ischemic stroke.

The cohort was restricted to manual workers, and further adjustment was made for socioeconomic subgroups as well as for population density in residential municipalities in order to reduce confounding from lifestyle related exposures and environmental air pollution. However, residual confounding cannot be ruled out and findings must be interpreted with caution.

Further studies are needed to confirm these findings and further explore the relationship between exposure to different types of particles and various doses and the occurrence of stroke.

\section{Acknowledgment}

The Swedish Council for Working Life and Social Research funded this study (2006-0849).

\section{References}

1. Stegmayr B, Asplund K. Ökad överlevnad i stroke men oförändrad risk att insjukna [Improved survival after stroke but unchanged incidence]. Swedish Medical Journal (Läkartidningen). 2003;100:3492-8 (English summary).

2. Medin J, Nordlund A, Ekberg K. Increasing stroke incidence in Sweden between 1989 and 2000 among persons aged 30 to 65 years: Evidence from the Swedish Hospital Discharge Register. Stroke. 2004;35:1047-51. http://dx.doi.org/10.1161/01. STR.0000125866.78674.96.

3. Zivin JA. Approaches to cerebrovascular diseases. In: Goldman L, Ausiello D, editors. Cecil Textbook of Medicine. 22nd ed. Philadelphia, PA, USA: Saunders; 2004:2280-98.

4. Kettunen J, Lanki T, Tiittanen P, Aalto PP, Koskentalo T, Kulmala M, et al. Associations of fine and ultrafine particulate air pollution with stroke mortality in an area of low air pollution levels. Stroke. 2007;38:918-22. http://dx.doi.org/10.1161/01. STR.0000257999.49706.3b. 
5. Miller KA, Siscovick DS, Sheppard L, Shepherd K, Sullivan $\mathrm{JH}$, Anderson GL, et al. Long-term exposure to air pollution and incidence of cardiovascular events in women. N Engl J Med. 2007;356:447-58. http://dx.doi.org/10.1056/NEJMoa054409.

6. Wellenius GA, Schwartz J, Mittleman MA. Air pollution and hospital admissions for ischemic and hemorrhagic stroke among medicare beneficiaries. Stroke. 2005;36:2549-53. http://dx.doi.org/10.1161/01.STR.0000189687.78760.47.

7. Notkola V, Pajunen A, Leino-Arjas P. Occupational mortality by cause in Finland 1971-91 and Occupational Mobility. Helsinki: Statistics Finland, Health 1997:1.

8. Rönneberg A. Mortality and cancer morbidity in workers from an aluminium smelter with prebaked carbon anodes - part III: Mortality from circulatory and respiratory diseases. Occup Environ Med. 1995;52:255-61. http://dx.doi.org/10.1136/ oem.52.4.255.

9. Coggon D, Wield G. Mortality of army cooks. Scand J Work Environ Health. 1993;19:85-8. http://dx.doi.org/10.5271/ sjweh.1493.

10. Torén K, Bergdahl IA, Nilsson T, Järvholm B. Occupational exposure to particulate air pollution and mortality due to ischaemic heart disease and cerebrovascular disease. Occup Environ Med. 2007;64:515-9. http://dx.doi.org/10.1136/ oem.2006.029488.

11. Nordisk yrkesklassificering [Nordic Occupational Classification]. Stockholm: Stockholm Labour Market Board; 1978.

12. Kauppinen T, Heikkilä P, Plato N, Woldbaek T, Lenvik $\mathrm{K}$, Hansen J, et al. Construction of job-exposure matrices for the Nordic Occupational Cancer Study (NOCCA). Acta Oncol. 2009;48:791-800. http://dx.doi. org/10.1080/02841860902718747.

13. Wiebert P. The impact of airway-irritating exposure and wet work on subjects with allergy or other sensitivity epidemiology and mechanisms. Stockholm: Department of Public Health Sciences, Karolinska Institutet; 2007.

14. Arbetsmiljöverket [Swedish Work Environment Authority]. Occupational exposure limit values and measures against air pollution [written ordinance]. AFS 2005:17; 27 December 2011. Available from www.av.se.

15. Kerr GD, Slavin H, Clark D, Coupar F, Langhorne P, Stott DJ. Do vascular risk factors explain the association between socioeconomic status and stroke incidence: A metaanalysis. Cerebrovasc Dis. 2011;31:57-63. http://dx.doi. org/10.1159/000320855.

16. Kuper H, Adami H-O, Theorell T, Weiderpass E. The socioeconomic gradient in the incidence of stroke: A prospective study in middle-aged women in Sweden. Stroke. 2007;38:2733. http://dx.doi.org/10.1161/01.STR.0000251805.47370.91.

17. McMichael AJ. Standardized mortality ratios and the "healthy worker effect": scratching beneath the surface. J Occup Med. 1976;18:165-8. http://dx.doi.org/10.1097/00043764197603000-00009.

18. The Swedish National Institute of Public Health. Health on Equal Terms - The National Survey of Public Health. Östersund (Sweden):The Swedish National Institute of Public Health; 2011.

19. Axelson O, Steenland K. Indirect methods of assessing the effects of tobacco use in occupational studies. Am J Ind Med 1988; 13: 105-18. http://dx.doi.org/10.1002/ ajim.4700130107.

20. Brook RD, Rajagopalan S, Pope CA, 3rd, Brook JR, Bhatnagar A, Diez-Roux AV, et al. American Heart Association Council on Epidemiology and Prevention, Council on the Kidney in Cardiovascular Disease, and Council on Nutrition, Physical Activity and Metabolism. Particulate matter air pollution and cardiovascular disease: an update to the scientific statement from the American Heart Association. Circulation. 2010;121:233178. http://dx.doi.org/10.1161/CIR.0b013e3181dbece1.

21. Chen LH, Knutsen SF, Shavlik D, Beeson WL, Petersen F, Ghamsary M, et al. The association between fatal coronary heart disease and ambient particulate air pollution: Are females at greater risk? Environ Health Perspect. 2005;113:1723-9. http://dx.doi.org/10.1289/ehp.8190.

22. Ibfelt E, Bonde JP, Hansen J. Exposure to metal welding fume particles and risk for cardiovascular disease in Denmark: A prospective cohort study. Occup Environ Med. 2010;67:772-7. http://dx.doi.org/10.1136/oem.2009.051086.

23. Toivanen S. Job control and the risk of incidental stroke in the working population in Sweden. Scand J Work Environ Health. 2008;34:40-7. http://dx.doi.org/10.5271/sjweh.1196.

24. Toivanen S, Hemström Ö. Is the impact of job control on stroke independent from socioeconomic status? A large-scale study of the Swedish working population. Stroke. 2008;39:1321-3. http://dx.doi.org/10.1161/STROKEAHA.107.495523.

25. Swedish Work Environment Authority. Arbetsmiljön 1999 [The work environment 1999]. AM 68 SM 0001. Stockholm: Swedish Work Environment Authority 2000:257. (English summary).

26. Virtanen SV, Notkola V. Socioeconomic inequalities in cardiovascular mortality and the role of work: A register study of Finnish men. Inter J Epidem. 2002;31:614-21. http:// dx.doi.org/10.1093/ije/31.3.614.

Received for publication: 17 August 2011 